ا و بـ- دانشجوى دكترى و استاديار، اصلاح نباتات، دانشكاه علوم كشاورزى و منابع طبيعى سارى

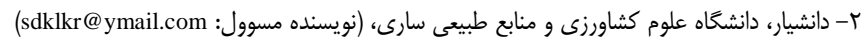

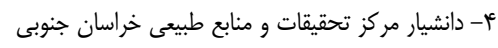

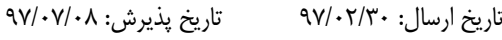

صفحه: وس تا بc

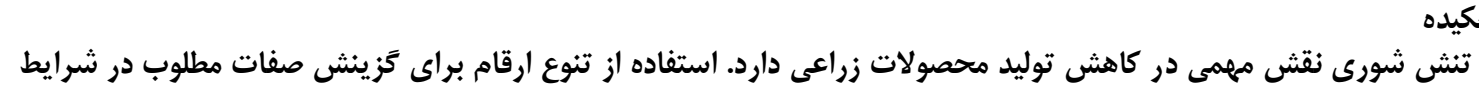

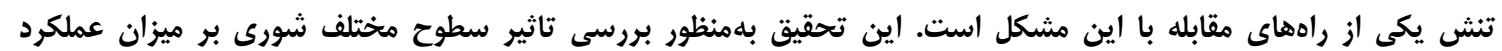

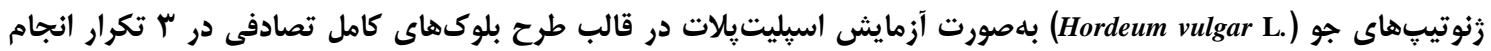

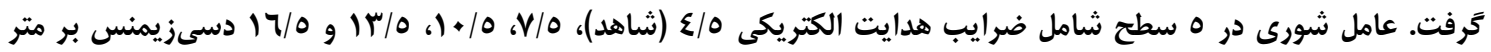

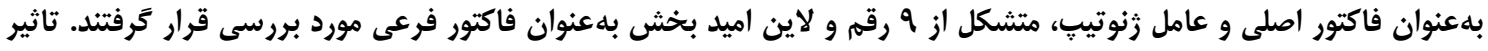

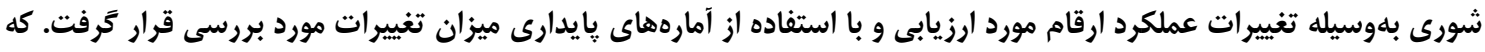

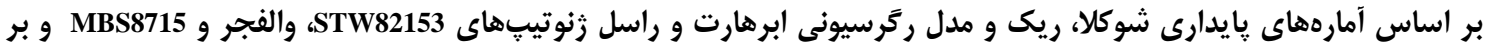

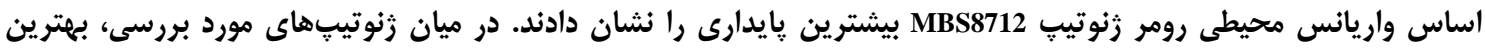

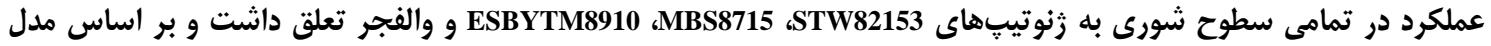

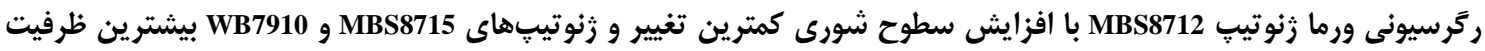

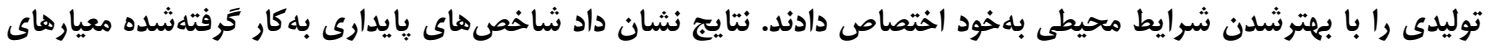

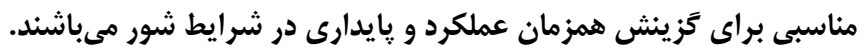
وازههاى كليدى: تنثى شورى، تجزيه پايدارى، جو، عملكرد

قبيل عملكرد بيولوزيك، متوسط دانه در سنبله، عملكرد كاه و

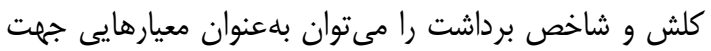

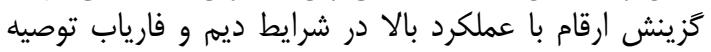

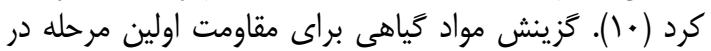

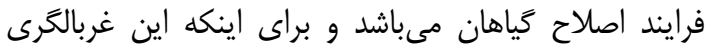

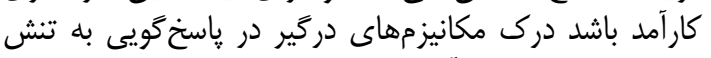

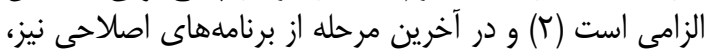

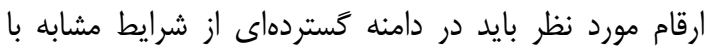

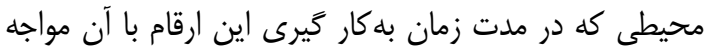

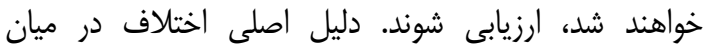

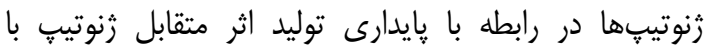

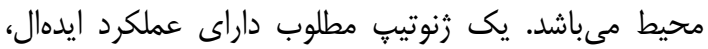

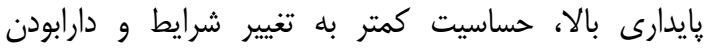

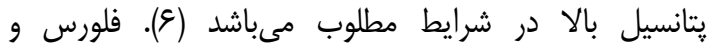

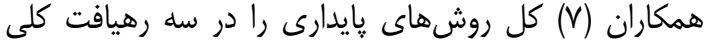

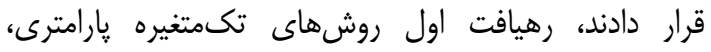

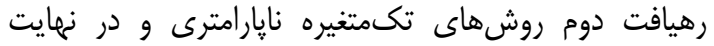

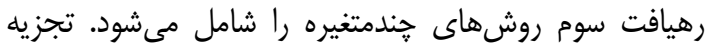

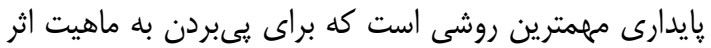

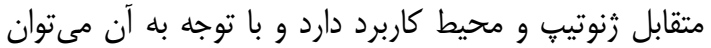

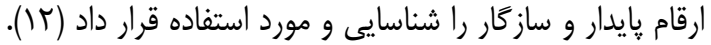

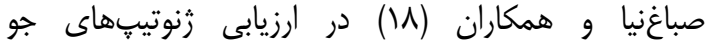

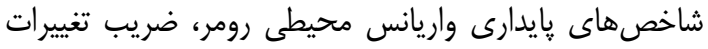

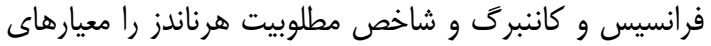

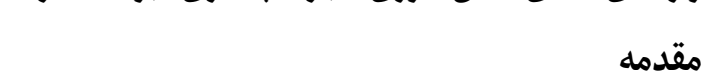

جو بهعنوان يك گياه زراعى سازگًار با شرايط ديم و متحمل

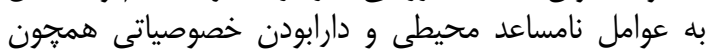

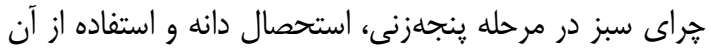

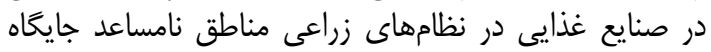

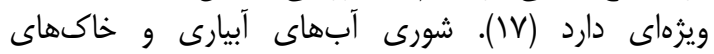

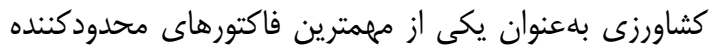
رشد محصولات زراعى و حاصلخيزى است و و واكنش كياهن مانهان

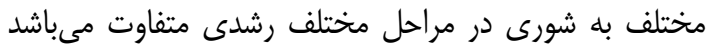

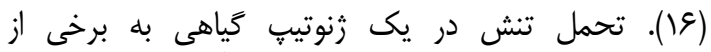

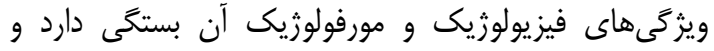

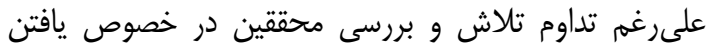

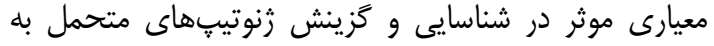

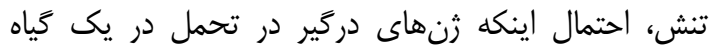

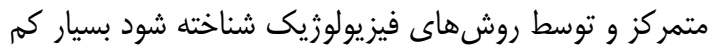

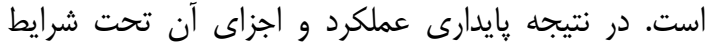

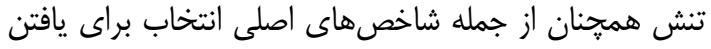

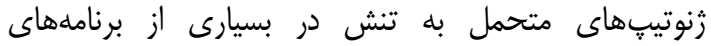

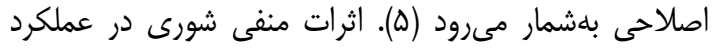
دانه جو از طريق كاهش تعداد ينجه بارور، تعداد سنبله، تعداد

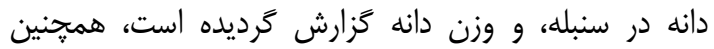

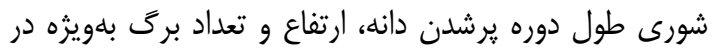

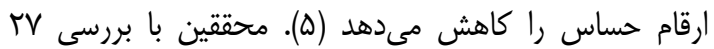
رنوتيب جو در شرايط ديم و فارياب نشان دادند خصوصياتى از 
$\mathrm{S}_{\mathrm{xi}}^{2}=\frac{\sum\left(\mathrm{x}_{\mathrm{ij}} \overline{\mathrm{x}}_{\mathrm{i} .}\right)^{2}}{(\mathrm{E}-1)}$

$\mathrm{w}_{\mathrm{i}}^{2}=\sum_{\mathrm{i}=1}^{\mathrm{n}}\left(\mathrm{x}_{\mathrm{ij}}-\overline{\mathrm{x}}_{\mathrm{i} \cdot} \cdot \overline{\mathrm{x}}_{\mathrm{j}}+\overline{\mathrm{x}}_{. .}\right)^{2}$

$\mathrm{b}_{\mathrm{i}}=1+\frac{\sum_{\mathrm{i}}\left(\mathrm{x}_{\mathrm{ij}}-\overline{\mathrm{x}}_{\mathrm{i} .}-\overline{\mathrm{x}}_{\mathrm{j}}+\overline{\mathrm{x}}_{. .}\right)\left(\overline{\mathrm{x}}_{\mathrm{j}}+\overline{\mathrm{x}}_{. .}\right)}{\sum_{\mathrm{j}}\left(\overline{\mathrm{x}}_{\mathrm{j}}+\overline{\mathrm{x}}_{. .}\right)^{2}}$

$S^{2}{ }_{d i}=\frac{1}{E-2}\left[\sum_{i}\left(x_{i j}-\bar{x}_{i .}-\bar{x}_{. j}+\bar{x}_{. .}\right)-\left(b_{i}-1\right)^{2} \sum_{j}\left(\bar{x}_{. j}+\bar{x}_{. .}\right)^{2}\right]$

$\sigma_{i}^{2}=\frac{G}{(E-1)(G-2)}$ wi- $\frac{\text { MsGE }}{\mathrm{G}-2}$

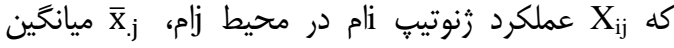

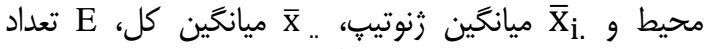

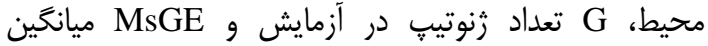

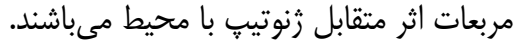

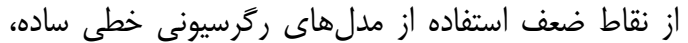

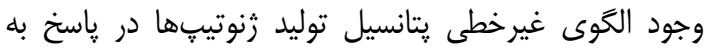

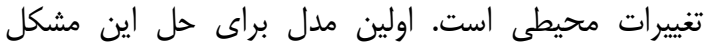

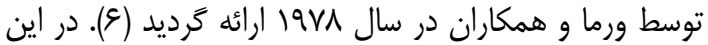

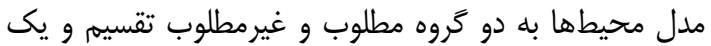

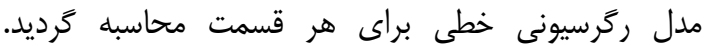
تفكيك محيطها بر اساس شاسئ شاخص محيطى كه ته تفاضل

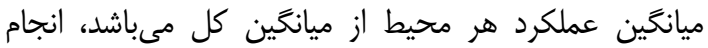

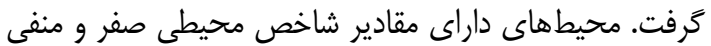

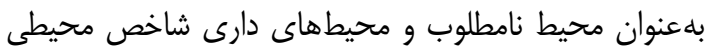

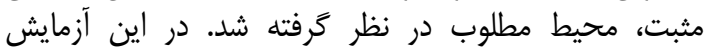

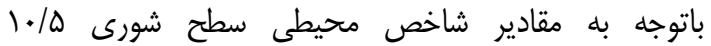

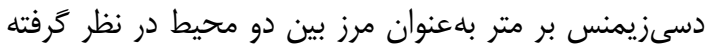

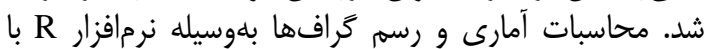

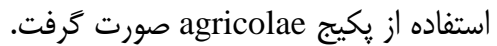

سطوح مختلف شورى اثر معنىدارى بر عملكرد زنوتيِها

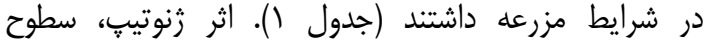

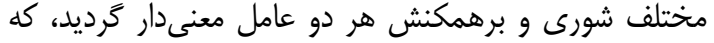

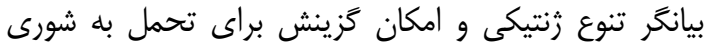

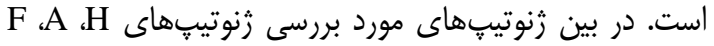

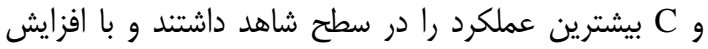

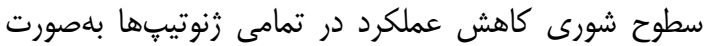

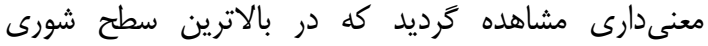

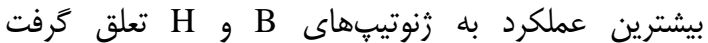

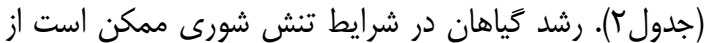

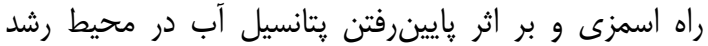

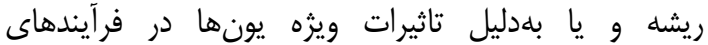

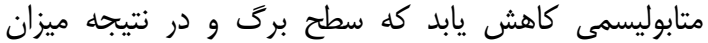
فتوسنتز در كل كياه كاهش خواهد كاهد يافت (9).
مناسبى جهت كزينش همزمان براى عملكرد و وِايدارى

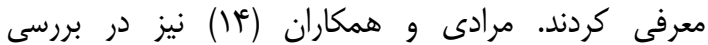

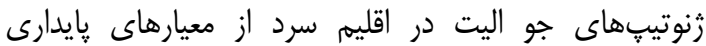

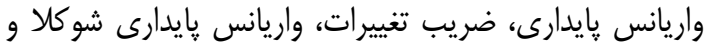

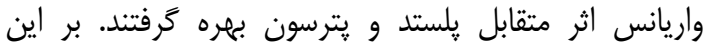

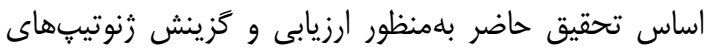

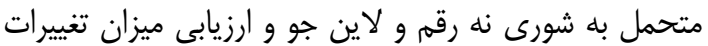
عملكرد آنها با استفاده از آمارههاى يايدارى انجام شار شد.

\section{مواد و روشها}

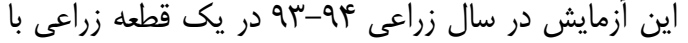

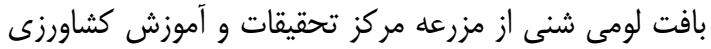

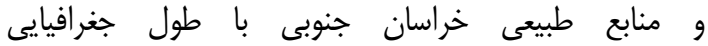

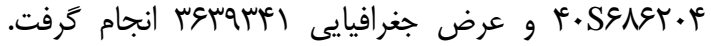

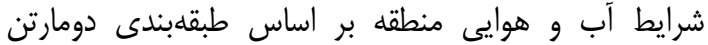

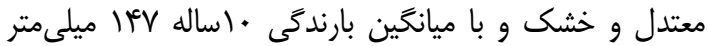

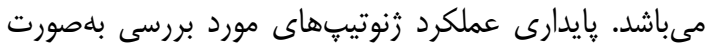

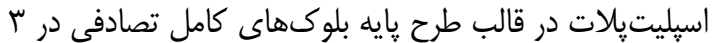

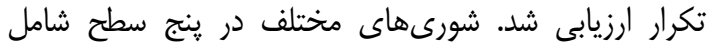

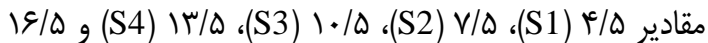

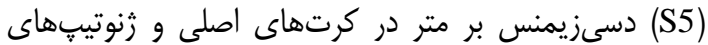

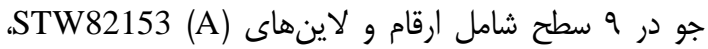

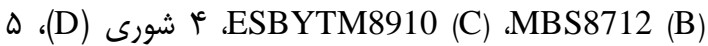

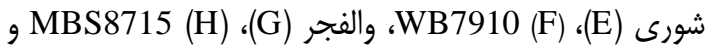
جو ترش (I) در كرتهاى فرعى قرار كرفتنند. از آنجا كه

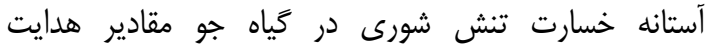

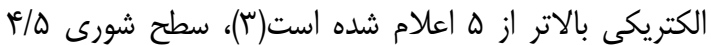

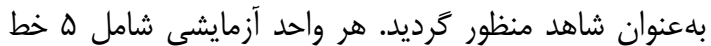

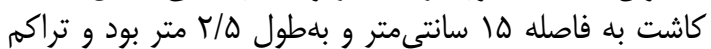

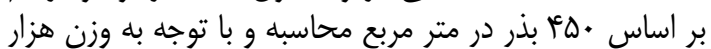

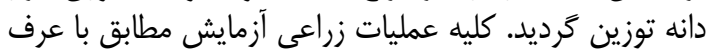

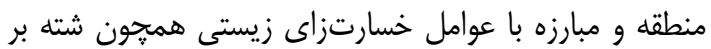

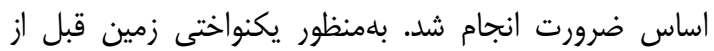

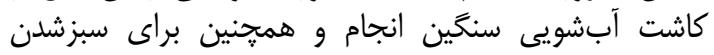

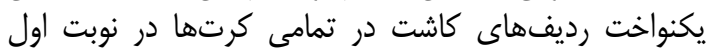

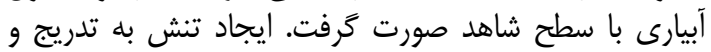

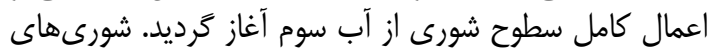

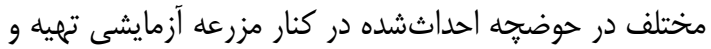

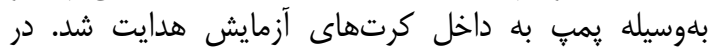

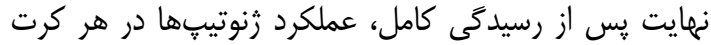

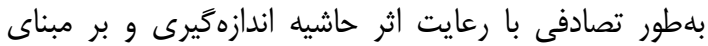

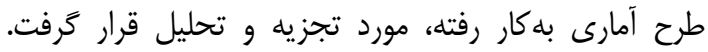

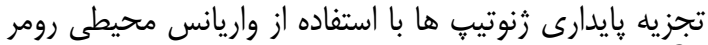

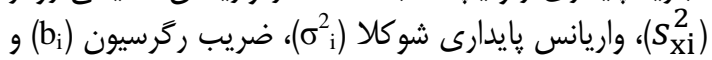

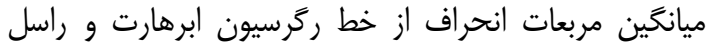

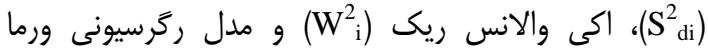
مطابق با فرمولهاى ذيل مورد بررسى قرار كرفتيند 


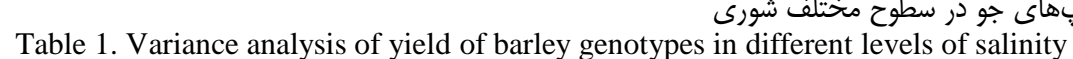

جدول ا- تجزيه واريانس عملكرد زنوتيیهاى جو در سطوح مختلف شورى

\begin{tabular}{|c|c|c|}
\hline ميانكين مربعات & درجه آزادى & منبع تغييرات \\
\hline & & يلات اصلى \\
\hline . & r & بلوى \\
\hline$\Delta D / \Delta^{* *}$ & i & شورى \\
\hline \multirow[t]{2}{*}{ ג } & $\wedge$ & خطاى اصلى \\
\hline & & هِات فرعى \\
\hline $1 / M^{* *}$ & $\wedge$ & رنوتيب \\
\hline ./M & r & رنوتيب × شورى \\
\hline.$/ \Delta f$ & ᄉ. & خطاى فرعى \\
\hline
\end{tabular}

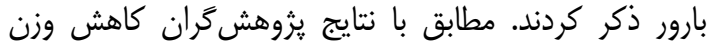

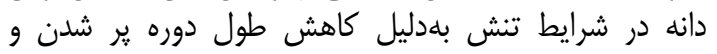

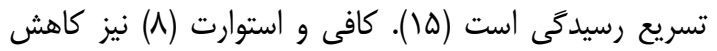
وزن دانه را در اثر تيمارهاى مختلف است شورى بـ بر عملكرد كَندم

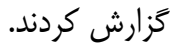

عدم تعادل يونى و وضعيت مواد غذايى در گياه تحت

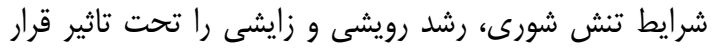

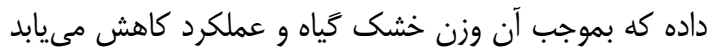

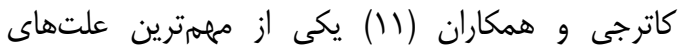
كاهش عملكرد در شرايط تنش شورى را كاهش تعداد ينجه

جدول r- مقايسه ميانكَين عملكرد زنوتيڤها در شر ايط تنش شورى با استفاده از روش دانكن در سطح احتمال ا درصد Table 2. Statistical comparison of means for yield (ton/ha) by Duncan's multiple range test ( $\alpha=0.01)$

\begin{tabular}{|c|c|c|c|c|c|c|}
\hline شنوتيّ & $\mathrm{S}_{1}$ & $\mathrm{~S}_{2}$ & $\mathrm{~S}_{3}$ & $\mathrm{~S}_{4}$ & $\mathrm{~S}_{5}$ & ميانكين رنوتيهها \\
\hline $\mathrm{A}$ & 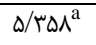 & $r / r r^{f g}$ & $r / \leftarrow \wedge v^{i}$ & $T / T \Lambda I^{\text {no }}$ & $1 / \mathrm{V} 10^{\mathrm{S}}$ & $r / F \mid r^{b c}$ \\
\hline B & $r / q 1 \Lambda^{c}$ & r/qน.h & $r / r r q^{j}$ & $r / r s)^{\text {no }}$ & 1/quv ${ }^{p q}$ & $\Gamma / T \wedge \Delta^{\mathrm{d}}$ \\
\hline $\mathrm{C}$ & $\Delta / \mu r \cdot{ }^{a}$ & $f / \Delta 1$. de & $\Gamma / \uparrow q \mu^{i}$ & $r / r r .{ }^{\text {no }}$ & $1 / v r q^{r s}$ & $r / \mathcal{q}{ }^{\mathrm{ab}}$ \\
\hline D & $\kappa / \varepsilon \Delta \cdot{ }^{\mathrm{d}}$ & h/Аrrh & $r / / f \gamma^{j k}$ & $r / \cdot \kappa^{F^{P}}$ & $y \cdot * \mu^{\text {tu }}$ & $r / q r q_{f}$ \\
\hline E & ५/१qभ & r/Tr/ & $r / v q .{ }^{m}$ & $\mathrm{~V} / \Lambda \propto \mathrm{v}^{\mathrm{pqrs}}$ & $V \cdot \cdot M^{\mathrm{tu}}$ & $T / \Delta q r^{g}$ \\
\hline $\mathrm{F}$ & $\Delta / \mu q \mu^{a}$ & $r / / r^{g}$ & $r / \cdot c \cdot{ }^{k l}$ & $(1 / 91)^{\mathrm{pq}}$ & $1 / r \cdot V^{s}$ & e \\
\hline G & $\Delta / I V{ }^{\mathrm{W}} \mathrm{b}$ & $r / r \ldots g$ & $r / \varangle \wedge v^{i}$ & $T / r \cdot 9^{\circ}$ & $1 / v \mid F^{t}$ & $\Gamma / \Gamma \Delta \mathcal{F}^{c d}$ \\
\hline $\mathrm{H}$ & $\Delta / \mathcal{F} \cdot \mu^{\mathrm{a}}$ & $r / r v .{ }^{e f}$ & $r / \leftarrow q \gamma^{\mathrm{i}}$ & $r / \& r I^{\mathrm{n}}$ & V/Aerqugs & $r / \Delta \cdot v^{\mathrm{a}}$ \\
\hline I & $r / \wedge \Delta v^{g}$ & $r / M \Lambda^{j \mathrm{k}}$ & r/MN"m & $V_{1 / q^{p q r}}$ & $V \cdot r \Lambda^{u}$ & $r / 91 D^{\mathrm{g}}$ \\
\hline شورى & $r / q \varphi^{a}$ & $r / q 9)^{b}$ & $\Gamma / r T \Delta^{c}$ & T/lFT & $1 / 4 V \Lambda^{e}$ & \\
\hline
\end{tabular}

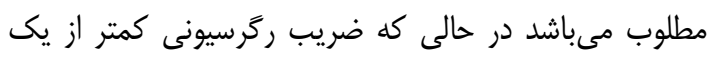

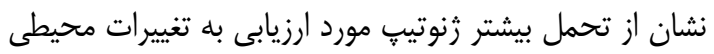

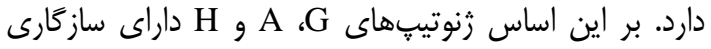

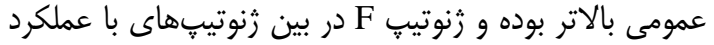

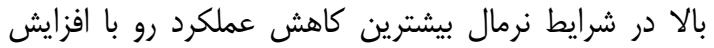

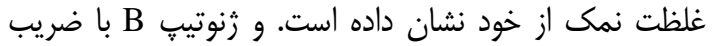

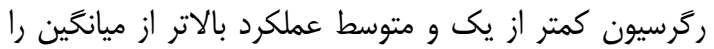

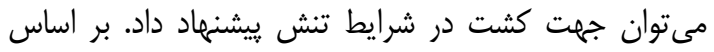

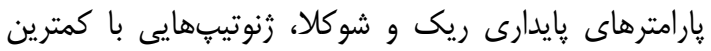

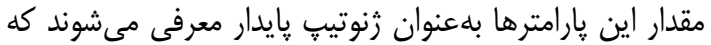

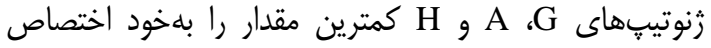

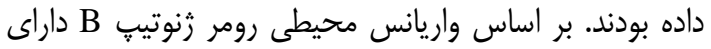

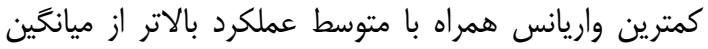

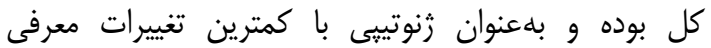
مى گر دد.
نتايج تجزيه واريانس نشان داد كه اثر متقابل ثنوتيڤ و دادي

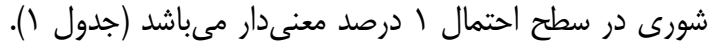

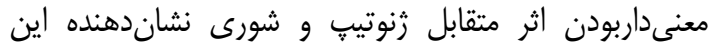

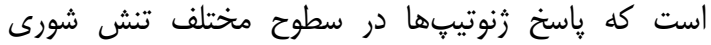

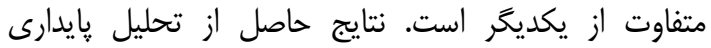

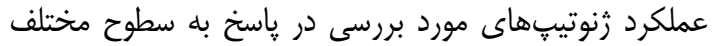

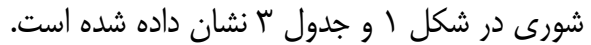

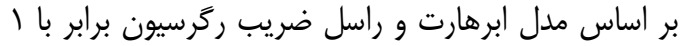

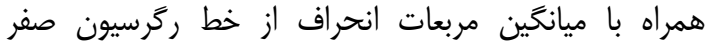

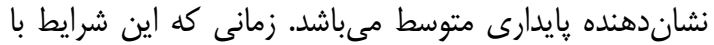

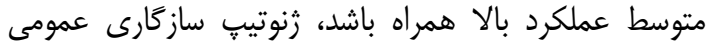

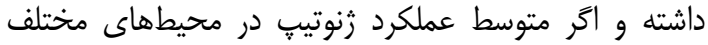

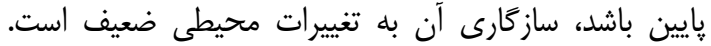

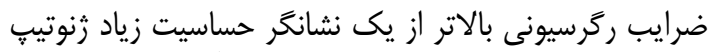

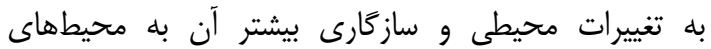



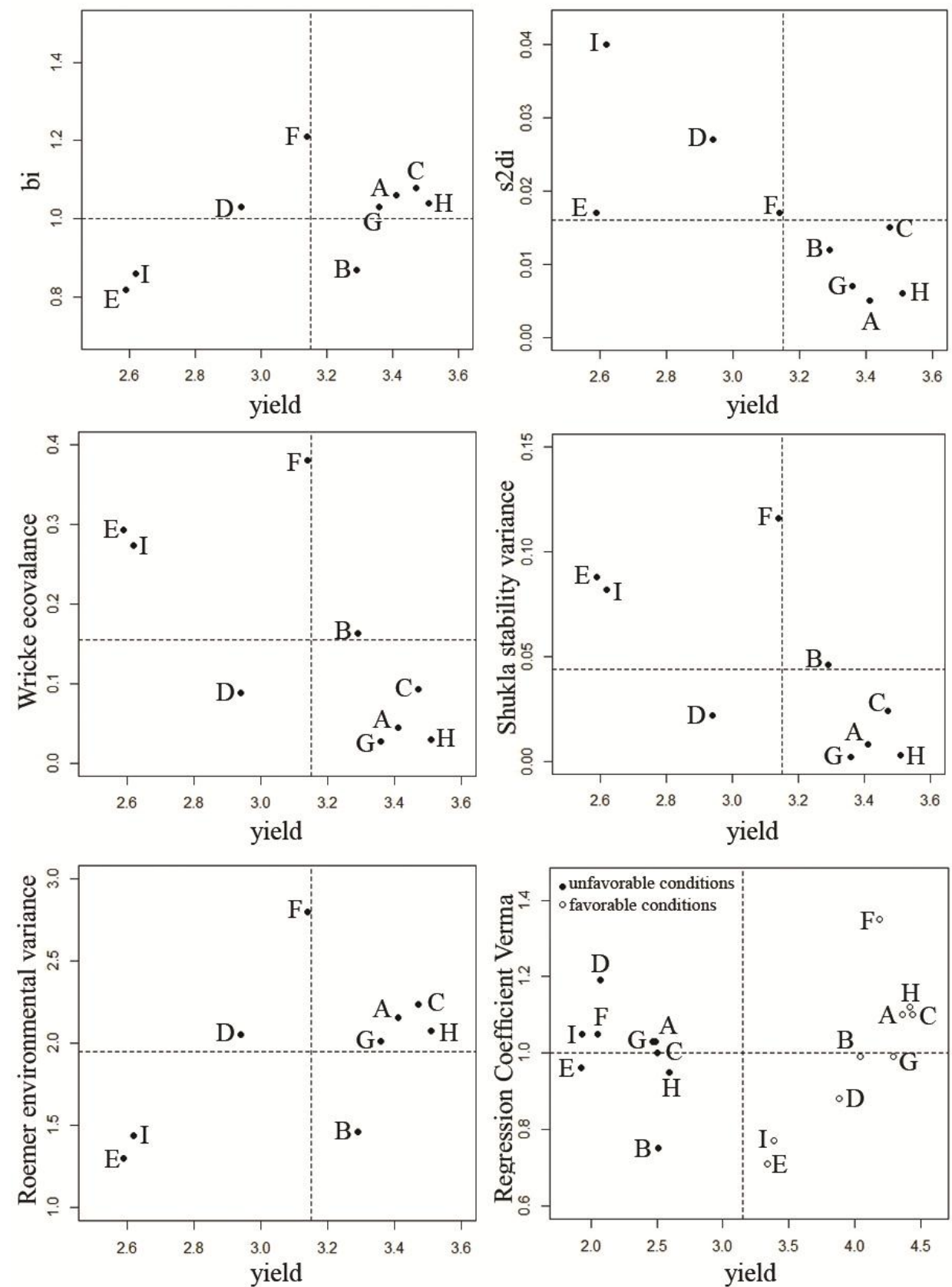

شكل ا - توزيع زنوتيِها بر اساس پارامترهاى پايدارى و عملكرد

Figure 1. Distribution of genotypes based on stability parameters and yield

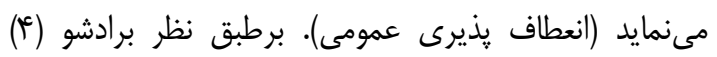

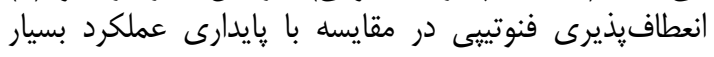

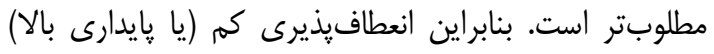

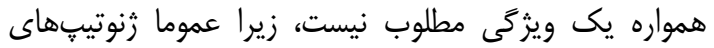

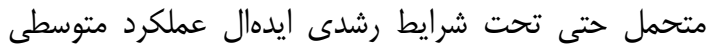

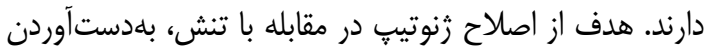

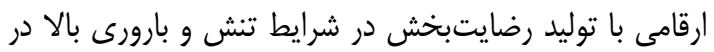

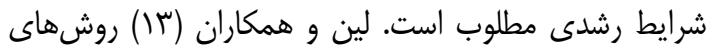

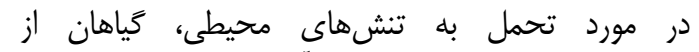

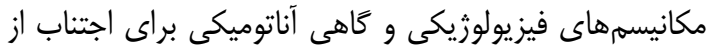

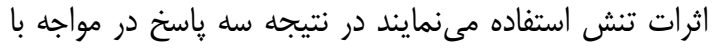

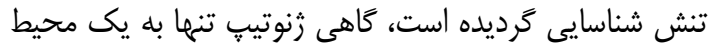

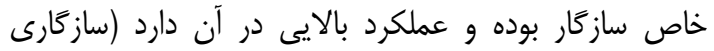

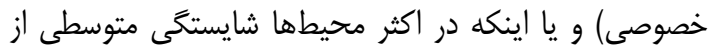

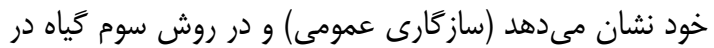

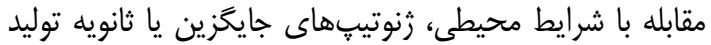


ميانخَين مربعات باقى مانده از مدل رگرسيونى روى شاخص

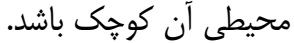

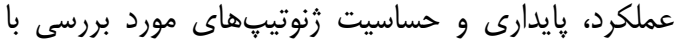

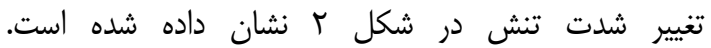

يارامترى پايدارى را به تيڤهاى مختلف تقسيهبندى كردند

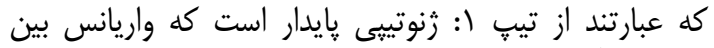

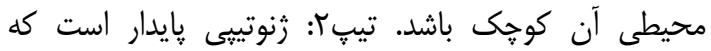

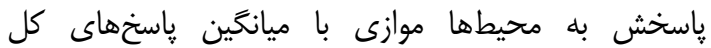

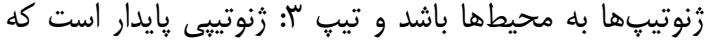

جدول س- يارامترهاى پايدارى زنوتيٍهاى جو بر اساس تغييرات عملكرد در شدتهاى متفاوت تنش شورى Table 3. Stability parameters of barley genotypes based on yield changes in different intensity of salinity stress

\begin{tabular}{|c|c|c|c|c|c|c|c|}
\hline \multirow{2}{*}{ رنوتي } & \multirow{2}{*}{$\mathbf{b}_{\mathbf{i}}$} & \multirow{2}{*}{$\mathrm{S}_{\mathrm{di}}^{2}$} & \multirow{2}{*}{$\mathbf{w}^{2}{ }_{i}$} & \multirow{2}{*}{$\sigma^{2}{ }_{i}$} & \multirow{2}{*}{$S_{x i}^{2}$} & \multicolumn{2}{|c|}{ ضريب رَّرسيونى ورما } \\
\hline & & & & & & محيط مطلوب & محيط غيرمطلوب \\
\hline A & $1 / .9$ & $\cdot / \cdot \Delta$ & .1 .44 &.$/ \cdots 1$ & $T / 1 Q$ & $1 / 1$ & $1 / r$ \\
\hline B & $\cdot /$ AV &.$/ .14$ & 更 & .1 .48 & $1 / \notin \Delta$ &.$/ 99$ & $\cdot / V \Delta$ \\
\hline $\mathrm{C}$ & $1 / \cdot 1$ & .1 .10 & . 1.94 &.$/ .4 t$ & سז/T & // & 1 \\
\hline D & $1 / \cdot r$ &.$/ \cdot t r$ &.$/ . M$ & . $1.4 t$ & $r / \cdot \Delta$ & $\cdot / M$ & $1 / 19$ \\
\hline E & . /Ar &.$/ .1 \mathrm{~V}$ & 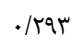 & $\cdot / \cdot M$ & $1 / r q$ & $\cdot / M 1$ & .198 \\
\hline $\mathrm{F}$ & $1 / r$ &.$/ \cdot \mathrm{lV}$ & 年 &.$/ 119$ & $r / v q$ & $1 / \pi \Delta$ & $1 / \cdot 0$ \\
\hline G & $1 / \cdot r$ & $\cdot / \cdot V$ & $/ .4 R$ & ...r & $r / \cdot 1$ &.$/ 99$ & 1.r \\
\hline $\mathrm{H}$ & $1 / \cdot \varphi^{c}$ & .1 .99 & $\cdot / \cdot r$ &.$/ . \mu$ & $r / \cdot V$ & $1 / 1 r$ &.$/ 90$ \\
\hline I & $\cdot / \Lambda \varepsilon$ & $\cdot / . e^{c}$ &.$/ T Y^{c}$ & . $/ . \wedge r$ & $1 / 4$ & $\cdot / V V$ & $1 / \cdot 0$ \\
\hline
\end{tabular}
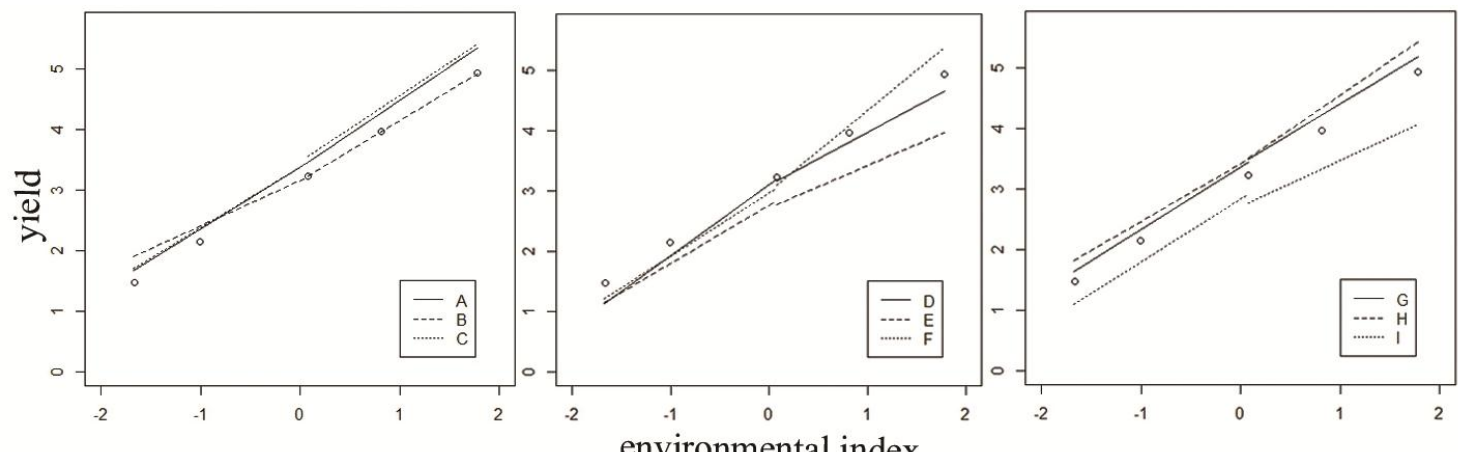

شكل r- عملكرد زنوتيبٍ هاى جو در سطوح مختلف شورى بر اساس مدل رگرسيونى ورما

Figure. 2. Performance of barley genotypes across salinity levels based on Verma regression model

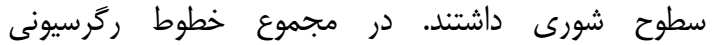

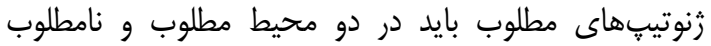

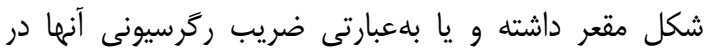

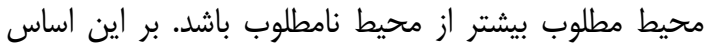

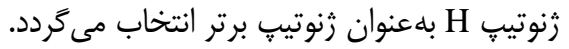

زنوتيههاى E و I عملكرد يايينترى نسبت به ميانگين

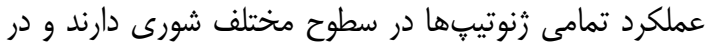

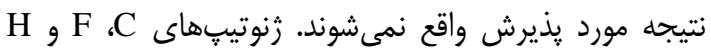

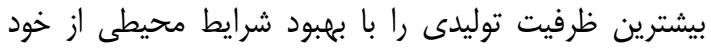

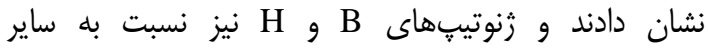

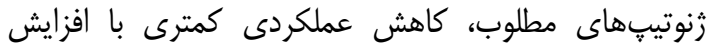


1. Adams, M.W. 1976. Basic of yield component compensation in crop with special reference to the field bean. Crop Science, 7: 505-510.

2. Adjel, F. and Z. Kadi. 2013. Salt stress Effects on seed germination and seedling growth of barley genotypes. Journal of Agriculture and sustainability, 3(2): 223-237.

3. Alizadeh, A. 1998. Quality of irrigation water. $5^{\text {th }}$ edition. Astan Ghods Razavi, IRAN, 96 pp. (In Persian).

4. Bradshaw, A.D. 2006. Unraveling phenotypic plasticity- why should we bother? N. Phytol, 170:644648.

5. Dadashi, M.R., I. Majidi Hervan, A. Soltani and A.A. Noorinia. 2006. Evaluation of different genotypes of barley to salinity stress. Journal of Agricultural Sciences, 1: 181-191 (In Persian).

6. Ferreira, D. and C. Demetrio. 2006. Statistical models in agriculture: biometrical methods for evaluating phenotypic stability in plant breeding. CERNE, 12: 373-388.

7. Flores, F., M.T. Moreno and J.I. Cubero. 1998. A comparison of univariate and multivariate methods to analyze G*E interaction. Field Crop Research, 56: 271-286.

8. Kafi, M. and D.A. Stwart. 1998. Effect of salinity on growth and yield nine types of wheat. Agronomy Food Science, 12(1): 77-85.

9. Kamrava, S., N. Babaeian Jelodar and N. Bagheri. 2016. Evaluation of Some Soybean Genotypes (Glycine max) under Salt Stress. Journal of Crop Breeding, 8(18): 57-63 (In Persian).

10. Karimi, E., M.R. Ghannadha, M.R. Naghavi and M. Mardi. 2005. An evaluation of drought resistance in barley. Iranian Journal of Agricultural Sciences, 36: 547-560 (In Persian).

11. Katerji, N., J.W. van Hoorn, A. Hamdy and M. Mastrorilli. 2006. Classification and salt tolerance analysis of barley varieties. Agriculture Water Management, 85: 84-92.

12. Koocheki, A.R. and M. Khodarahmi. 2013. Phenotypic plasticity analysis of promising barley genotypes in cold zone of Iran. Journal of Crop Production Research, 4: 321-332 (In Persian).

13. Lin, C.S., M.R. Binns and L.P. Lefcovitch. 1986. Stability analysis: where do we stand? Crop Science, 26: 894-900.

14. Moradi, M., H. Dehghani and B. Sorkhi-Lalelo. 2012. Study of stability parameters in barley (Hordeum vulgar L.) elite genotypes in cold climate of Iran. Iranian Journal of Field Crop Research, 10: 107-115 (In Persian).

15. Naseer, S.H. 2001. Response of barley (Hordeum vulgar L.) at various stages to salt stress. Journal of Biological Science, 1(5): 326-329.

16. Niazian, M., M. Nomani and S.A. Sadat Noori. 2016. A Review on Biometrical Methods used for Salt Tolerance Breeding in Crops. Journal of Crop Breeding, 8(17): 21-41 (In Persian).

17. Rezaii kalo, S., M. Khodarahmi and Kh. Mostafavi. 2012. Study of traits in different barley types using factor analysis under terminal drought stress and without stress conditions. Journal of Agronomy and Plant Breeding, 3: 149-160 (In Persian).

18. Sabaghnia, N., M. Mohammadi and R. Karimizadeh. 2013. Yield stability of performance in multienvironment trials of barley (Hordeum Vulgar L.) Genotypes. Acta Universitatis Agriculturae Et Silviculturae Mendelianae Brunensis, 87: 787-793. 


\title{
Evaluation of Yield of Barley Genotypes (Hordeum Volgar L.) under Salinity Stress by Using Stability Analysis Methods
}

\section{Hossein Askari ${ }^{1}$, Syed Kamal Kazemitabar ${ }^{2}$, Hamid Najafi Zarrini ${ }^{3}$ and Mohammd Hossein Saberi ${ }^{4}$}

\author{
1 and 3- Ph.D. Student and Assistant Professor, Sari Agricultural Sciences and Natural Resources University \\ 2- Associate Professor, Sari Agricultural Sciences and Natural Resources University \\ (Corresponding author:sdklkr@ymail.com) \\ 4- Associate Professor, Provincial Research Center of Khorasan Jonobi \\ Received: May 20, $2018 \quad$ Accepted: September 30, 2018
}

\begin{abstract}
Salinity stress has an important role in reducing crop production. There is a possibility of cultivar diversity use in desirable traits selection under stress. This research was carried out in order to test of different salinity level on yield of barley (Hordeum Volgar L.) genotypes, in the split plot experiment on the base of Randomized Completely Block design in 3 replications. Salinity treatment involved 5 levels: $S_{1}$ (control)=EC $4.5 \mathrm{ds} / \mathrm{m}, \mathrm{S}_{2}=E C 7.5 \mathrm{ds} / \mathrm{m}, \mathrm{S}_{3}=\mathrm{EC} 10.5$ $\mathrm{ds} / \mathrm{m}, \mathrm{S}_{4}=\mathrm{EC} 13.5 \mathrm{ds} / \mathrm{m}$ and $\mathrm{S}_{5}=\mathrm{EC} 16.5 \mathrm{ds} / \mathrm{m}$ as main plot and subplots were 9 genotype involved promising lines and varieties. The effects of Salinity treatments studied by sampling on yield of genotypes and using stability parameters. That based on Shukla stability variance, Wricke ecovalance and Eberhart and Russell regression model the STW82153, MBS8715 and Valfajr genotypes and based on Romero environmental variance the MBS8712 genotype exhibited the most stability. The best performance in all salinity levels was shown by genotypes STW82153, MBS8715, ESBYTM8910 and Valfajr. Genotype MBS8712 showed the least change with increasing salt concentration and with the improvement of environmental conditions, genotypes MBS8715 and WB7910 had the highest production capacity. The results indicated that used stability parameters would be useful for simultaneously selecting for high yield and stability under salinity conditions.
\end{abstract}

Keywords: Barley, Salinity stress, Stability parameters, Yield 